\title{
PHASE BEHAVIOUR OF HIGH, MEDIUM AND LOW MELTING FRACTIONS IN LAURIC FAT BLENDS FORMULATED BASED ON MELTING TRIACYLGLYCEROL GROUP
}

\section{BANGUN NUSANTORO*; YANTY NOORZIANNA MANAF**; DAVY VAN DE WALLE*; FILIP VAN BOCKSTAELE* and KOEN DEWETTINCK*}

\begin{abstract}
Lauric fat blends aimed for bakery shortening were prepared as a ternary mixture characterised by three distinct melting peaks on their differential scanning calorimeter (DSC) melting thermograms. Those melting peaks closely correlated to the high, medium, and low melting fractions (HMF, MMF and LMF) in the blends. In the simpler binary mixture, hard fats, (i.e. palm stearin and fully hydrogenated rapeseed oil) formed an ideal solubility in different liquid oils [i.e. medium chain triacylglycerol oil (MCT-oil) and high oleic sunflower oil (HOSO)]. However, slight deviations from ideality were observed when liquid oils were replaced by lauric oils (i.e. palm kernel oil and coconut oil). In the more complex ternary mixture, the HMF formed an ideal solubility in both the MMF and LMF within the range of given formulas. In addition, the interaction of solid crystals in lauric fat blends was of monotectic behaviour as shown in the isosolid diagram. Nevertheless, the influence of solubility seemed to override that of solid interaction. Both the solubility curves and isosolid lines can provide valuable information about the interaction of HMF, MMF and LMF in lauric fat blends. Therefore, they are useful for improving the formulation of plastic fats.
\end{abstract}

Keywords: fat blend, isosolid, lauric, phase behaviour, solubility, tag group.

Received: 12 May 2019; Accepted: 5 August 2020; Published online: 5 October 2020.

\section{INTRODUCTION}

Lauric fat blends in this research are a ternary mixture of a lauric oil [i.e. palm kernel oil (PKO) or coconut oil (CO)] with two other vegetable oils and fats prepared for the production of plastic fats (e.g. shortening, margarine and fat spread). Lauric fat blends can be formulated based on melting triacylglycerol (TAG) group (Nusantoro et al., 2017),

\footnotetext{
Laboratory of Food Technology and Engineering, Department of Food Technology, Safety and Health, Faculty of Bioscience Engineering,

Ghent University, Belgium.

E-mail: bpnusantoro@ugm.ac.id

** Halal Products Research Institute, Universiti Putra Malaysia, Putra Infoport, 43400 UPM Serdang, Selangor, Malaysia.
}

enabling some adjustments on the solid fat content (SFC) at temperature intervals particularly from $0^{\circ} \mathrm{C}-20^{\circ} \mathrm{C}$ and above $20^{\circ} \mathrm{C}$ (Nusantoro et al., 2013) to have the required SFC profiles for bakery shortening production. Lauric oils are mainly composed of fully saturated medium-chain fatty acids. The TAG derived from this type of fatty acids serve as the main source of medium melting TAG. These medium melting components play an important role in developing the key functionalities of plastic fats such as structure, mechanical performance and oral properties (Chai et al., 2018; Liu et al., 2018).

In order to get good plasticity, both solid and liquid phases should be present in a proper proportion in the fat blends (Nusantoro et al., 2016; Yanty et al., 2017). Liquid oils comprise entirely of low melting components and contribute to the 
major part of the blends as the liquid phase. In addition, some amount of hard fats is added as they are required in the blends to provide high melting components that give desired appearance, texture, and mouthfeel properties (O'Brien, 2009). Medium chain triacylglycerol oil (MCT-oil) and high oleic sunflower oil (HOSO) are examples of the liquid oils while palm stearin (PS) and fully hydrogenated rapeseed oil (FHRO) are those of the hard fats. Fat and oil sources for the fat blends can thus, come naturally or from fat modification.

The study of the phase behaviour of fat blends can lead to a better understanding of the interactions, occurring among the blend constituents and the various phase changes of their physical state. The knowledge of phase behaviour is of particular importance in the prediction of fat and fat blend properties, control of physical attributes, process optimisations and eventually product quality maintenance (Timms, 1984; Saberi et al., 2011). The formulated lauric fat blends have three distinct fractions differentiated by the melting point of their TAG compositions. The mixing behaviour of higher melting fractions in lower melting fractions directly relates to crystallisation behaviour of the fat blends. Moreover, there is a softening effect of LMF due to the dissolution of high-melting TAG (solute) into low-melting TAG (solvent). A solubility curve of fat blends can then be constructed to show equilibrium relationships between the temperature and the composition of solid fat in liquid oil (Zhou and Hartel, 2006).

When lower melting components do not involve in crystallisation, fat blends form an ideal solution and the solubility of high-melting TAG in low melting TAG, which follows the Hildebrand equation (Equation 1).

$$
\operatorname{Ln} x_{s}=\frac{\Delta H_{f}}{R}\left(\frac{1}{T_{m}}-\frac{1}{T}\right)
$$

Equation (1)

where $x_{s}$ is the solubility of high-melting component $\left(\right.$ mole mole $\left.{ }^{-1}\right), \Delta H_{f}$ is the heat of fusion of high-melting component $\left(\mathrm{J} \mathrm{mol}^{-1}\right), \quad R$ is the universal gas constant $\left(8.314 \mathrm{~J} \mathrm{~mol}^{-1} \mathrm{~K}^{-1}\right)$, and $T_{m}$ and $T$ are melting temperatures of the high-melting component and the mixture $(\mathrm{K})$, respectively. Although there are various methods to determine the melting temperature, the most preferred and widely used method is by using the differential scanning calorimeter (DSC). In the occurrence of some interactions among fat constituents, deviations from the ideal solution behaviour can be seen in a solubility curve (Walstra, 2003).

Lauric fat blends are a multicomponent system of TAG mixtures. In simpler binary TAG mixtures, phase behaviour of the blends can be either monotectic, eutectic or peritectic depending on their molecular interaction. Moreover, physical classification of TAG can be utilised as a method to reduce the complexity of TAG mixtures. TAG species are sorted into TAG categories that are supposed to form distinct separate solid fractions in the crystallised fat blend. In lauric fat blends, classification of TAG is based on the melting point of which four categories are formed namely heated $(\mathrm{H})$, body $(\mathrm{B})$, room $(\mathrm{R})$ and cool $(\mathrm{C})$ melting TAG groups. These groups represent the high, medium and low melting components in the fat blends (Wesdorp et al., 2005; O'Brien, 2009). Eventually, an isosolid diagram can then be constructed to investigate the type of solid solution behaviour of lauric fat blends. This pseudo-phase diagram shows lines of constant SFC at different temperatures (vertical axis) and composition (horizontal axis). For example, the isosolid line is commonly found to be a straight line parallel to the horizontal axis or to one of the lateral axes in the monotectic mixture. A eutectic mixture, on the other hand, exhibits a compression of the isosolid line which shows a constant SFC and a melting temperature below that of the individual fat. In the eutectic system, a considerable decrease of the SFC followed by softening of the fat blend is commonly observed (Timms, 1984; Zhou et al., 2010).

In this study, the complex interaction of high, medium and low melting fractions (HMF, MMF and LMF) in lauric fat blends was investigated. The solubility of hard fats in either lauric or liquid oils was firstly observed by solubility diagram in a simpler system as binary mixtures. Then, the application of Hildebrand equation was extended to evaluate the solubility of both HMF in lower melting components and HMF/MMF in LMF as a ternary mixture of lauric fat blends. Accordingly, the isosolid diagram was constructed for lauric fat blend at different formulations. The melting group TAG formulation was employed to give different ratios of HMF to MMF as isosolid lines which can evaluate such interaction of both fractions. Any changes and deviations from ideality of both the solubility curves and isosolid lines were indicated in order to provide useful guidance for developing plastic fat products.

\section{MATERIALS AND METHOD}

\section{Materials}

All vegetable oils and fats are commercial products as follows: Neobee M5 as the MCT-oil (Stepan, USA), HOSO (Oleon, Belgium), PS and PKO (Loders Crocklaan, The Netherland), coconut oil (Palko Sari Eka, Indonesia) and fully hydrogenated rapeseed oil (Palsgaard, Denmark). All other chemicals and solvents for analysis are those of the high purity grade. 


\section{The Binary Mixtures and Lauric Fat Blends}

In the first part of the investigation, six binary blends were prepared from two types of hard fats (PS and FHRO) and three types of either lauric or liquid oils (PKO, HOSO and MCT-oil) at different molar ratios of the high melting fraction from 1 to 0 with an interval of 0.1 mole mole ${ }^{-1}$. This simpler binary model of fat blends was used to study the ideal solubility of hard fats in lower melting components. The outcomes were then utilised to study the pseudo-phase behaviour of more complex ternary mixture of lauric fat blends.

Lauric fat blends were formulated based on the grouping of various TAG species according to their melting points $(\mathrm{mp})$. The TAG were grouped into four namely chilled (C) $\left(\mathrm{mp} \leq 5^{\circ} \mathrm{C}\right)$, room $(\mathrm{R})\left(5^{\circ} \mathrm{C}<\right.$ $\left.\mathrm{mp}<25^{\circ} \mathrm{C}\right)$, body $(\mathrm{B})\left(25^{\circ} \mathrm{C}<\mathrm{mp}<45^{\circ} \mathrm{C}\right)$ and heated (H) $\left(45^{\circ} \mathrm{C} \leq \mathrm{mp}\right)$ melting TAG groups. For example, a formulation of $\mathrm{BH} 50 \mathrm{H} 15$ indicated that the sample consisted of 50\% TAG from a combination of B and $\mathrm{H}$ groups and 15\% TAG from $\mathrm{H}$ group (thus, $\mathrm{B}$ group had 35\% TAG). The other 50\% of TAG in the formulation then came from a combination of $\mathrm{C}$ and R groups (Nusantoro et al., 2017).

In the second part of the study, two formulations of lauric fat blends were chosen. First, lauric fat blends were prepared from mixtures of $\mathrm{FHRO}, \mathrm{CO}$, and HOSO. The blends were formulated at constant $\mathrm{BH} 50$ and varied $\mathrm{H}$ from $2.5 \%-17.5 \%$ (seven blends, BH50 - H: 2.5/5/7.5/10/12.5/15/17.5). Second, lauric fat blends were prepared from FHRO, CO, and HOSO. The blends were formulated at constant $\mathrm{H} 15$ and varied $\mathrm{BH}$ from $50 \%-70 \%$ (five blends, H15 - BH: 50/55/60/65/70). Hildebrand plots and isosolid diagrams were then constructed from these formulated blends.

\section{Fatty Acid Composition}

The fatty acid composition of the samples was determined by gas chromatography (GC) after conversion to fatty acid methyl esters (FAME) using $2 \mathrm{~N} \mathrm{KOH}$ in methanol solution. The FAME were analysed by a Thermo Focus GC (Interscience, Belgium) equipped with an RTX-2330 column (cyanopropyl polysiloxane, $60 \mathrm{~m}$ length, $0.25 \mathrm{~mm}$ internal diameter, $0.2 \mu \mathrm{m}$ layer thickness) and a flame ionisation detector (FID). FAME were identified by comparing their retention time to authentic standards. Fatty acid levels were reported as relative proportions of the total composition.

\section{TAG Distribution}

The TAG distribution based on the equal carbon number was determined by non-aqueous reversedphase high-performance liquid chromatography (NARP-HPLC). The chromatographic system consisted of a Shimadzu HPLC system (Shimadzu, Japan) fitted with a $150 \times 3.0 \mathrm{~mm}$ Alltima HP C18-HL column (Grace Alltech, Belgium), and the detection was done by an Alltech 3300 ELSD (Grace Alltech, Belgium). An optimised method as previously described (Rombaut et al., 2009) was used for the measurement. Elution was carried out using an acetonitrile/dichloromethane gradient at $0.72 \mathrm{ml}$ $\mathrm{min}^{-1}$. The result was processed by LC solution software (Shimadzu, Japan).

\section{Melting Behaviour by DSC}

Thermal analyses of the samples were performed on a TA Q1000 DSC (TA Instruments, USA) equipped with a refrigerated cooling system. Nitrogen was used to purge the system. The samples (5-15 mg) were hermetically sealed in aluminium pans. An empty pan was used as a reference during the measurements. The time-temperature program of the measurements was as follows: holding at $90^{\circ} \mathrm{C}$ for $10 \mathrm{~min}$, cooling at a rate of $5^{\circ} \mathrm{C} \mathrm{min}{ }^{-1}$ to a temperature of $-70^{\circ} \mathrm{C}$, holding for $10 \mathrm{~min}$ at that temperature to allow complete crystallisation, and then heating at a rate of $5^{\circ} \mathrm{C} \mathrm{min}^{-1}$ to $90^{\circ} \mathrm{C}$. The obtained data were processed by the TA Universal Analysis 2000 software (TA Instruments, USA).

\section{SFC by pNMR}

SFC was measured according to the official IUPAC method by a Maran Ultra NMR (Oxford Instruments, United Kingdom) equipped with R4 cryostat cooler (Grant Instruments, United Kingdom). NMR tubes (Bruker, Germany) were used for the direct SFC measurement. The samples were prepared by the stabilising fat procedure where a tempering stage was included at $26^{\circ} \mathrm{C}$ for $40 \mathrm{hr}$ and subsequently at $0^{\circ} \mathrm{C}$ for $90 \mathrm{~min}$. The SFC values were then recorded at $5^{\circ} \mathrm{C}$ intervals from $0^{\circ} \mathrm{C}-60^{\circ} \mathrm{C}$. Before each reading, the samples were conditioned for $60 \mathrm{~min}$ at that chosen measurement temperature.

\section{Determination of Solubility and Isosolid Diagrams}

The solubility of the fat blends was depicted by solubility curves showing the equilibrium relationship between temperature and composition of higher melting components in lower melting components. The Hildebrand equation (Equation 1) was used in the calculation to construct the solubility diagram of which the melting point of samples was measured by DSC. Meanwhile, the SFC data (by p-NMR measurement) was used to construct the isosolid diagram following a procedure as previously reported (Timms, 1979). The solid interaction of the fat blends is then described by isosolid lines that connect points with a common SFC value. 


\section{Texture Analysis}

Texture analysis was carried out using a universal testing machine Instron 5942 (Instron, USA). A constant speed penetration was used to measure the samples as previously described (Braipson-Danthine and Deroanne, 2004). Prior to analysis, approx. A $50 \mathrm{~g}$ lauric fat blends were completely melted at $90^{\circ} \mathrm{C}$ for $10 \mathrm{~min}$ and then stored in a thermostatic cabinet at $15 \pm 1^{\circ} \mathrm{C}$ for $24 \mathrm{hr}$. Four penetration tests were run on each fat blend and the data were processed by the Bluehill 3 software (Instron).

\section{RESULTS AND DISCUSSION}

\section{Chemical Composition of Starting Oils and Fats}

The fatty acid composition of starting oils and fats is presented in Table 1. Lauric oils, such as coconut oil and palm kernel oil, characterised by a high content of medium-chain saturated fatty acids namely lauric (48.90\%-50.49\%) and myristic $(15.46 \%$ $17.70 \%$ ) acids. High oleic sunflower oil is composed mainly of long-chain monounsaturated fatty acid, namely oleic acid $(85.13 \%)$. Meanwhile, palm stearin and fully hydrogenated rapeseed oil consisted of long-chain saturated fatty acids with palmitic $(82.19 \%)$ and stearic $(89.77 \%)$ acids as their major constituents, respectively. The TAG distribution of starting oils and fats was also found to be close to the expected proportions of the relevant fatty acids as indicated in Table 2. When TAG were sorted based on their melting points, a handful of variables with easier physical interpretation can be obtained. It was shown that high, medium, and low melting fractions were closely related to the categorisation of TAG in the fat blends without neglecting the nature of starting oils and fats. The LMF was associated with both $C$ and $R$ groups while the MMF and HMF were associated with $\mathrm{B}$ and $\mathrm{H}$ groups, respectively.

\section{Solubility of the Simpler Binary System}

Melting properties of hard fats in the binary mixtures. A pure hard-melting fat which is composed of only a few TAG species usually melts within a very narrow temperature range. Moreover, the hard fat with longer fatty acid chain-length has a higher melting point and molar enthalpy of fusion compared to the one with shorter fatty acid chain length (Zhou and Hartel, 2006). Figures 1 and 2 showed the DSC melting thermograms of binary mixtures of hard fats (PS or FHRO) in medium melting fat (PKO) or low melting oils (HOSO or MCT-oil) at different molar ratios of the high melting fraction. Palm stearin melted completely at a lower temperature compared to $\mathrm{FHRO}$ in different types of solvents (PKO, HOSO, or MCToil) at all molar ratios. The softening effect of the solvents was also observed on which the melting point of the hard fats was depressed followed their molar ratios in the binary mixtures. For example, the peak maximum of PS and FHRO in HOSO was decreased from $61.08^{\circ} \mathrm{C}$ and $68.26^{\circ} \mathrm{C}$ to $48.93^{\circ} \mathrm{C}$ and $57.42^{\circ} \mathrm{C}$, at 0.7 and 0.1 molar of high melting fraction, respectively. This depressed melting point of hard fats plays an important role in the formulation of plastic fats as their high melting components can result in unwanted pasty and waxy mouthfeel. The HMF is known to have a tendency to adhere to palate, teeth and tongue (Drewnowski and Almiron-Roig, 2010; Podchong et al., 2020). Higher SFC of fat blends is commonly achieved by the addition of hard fats but higher molar ratios of the high melting fraction can significantly increase the melting point of the products. Therefore, the amount of hard fat should be carefully calculated to avoid undesired textural and sensorial properties, yet still giving appropriate functional attributes to the system such as structure, stabiliser, and moisture barrier (O'Brien, 2009; Danthine, 2012; Nusantoro et al., 2016).

TABLE 1. FATTY ACID COMPOSITION OF STARTING OILS AND FATS

\begin{tabular}{lccccc}
\hline \multirow{2}{*}{ Fatty acid } & \multicolumn{5}{c}{ Samples } \\
\cline { 2 - 6 } & HOSO (\%) & CO $(\%)$ & PKO $(\%)$ & PS (\%) & FHRO (\%) \\
\hline Caprylic (C8:0) & - & 8.59 & 4.07 & - & - \\
Capric (C10:0) & - & 6.62 & 4.06 & - & - \\
Lauric (C12:0) & - & 48.90 & 50.49 & - & - \\
Myristic (C14:0) & - & 17.70 & 15.46 & 1.31 & - \\
Palmitic (C16:0) & 3.95 & 8.26 & 7.81 & 82.19 & 6.97 \\
Stearic (C18:0) & 3.17 & 2.44 & 2.13 & 4.73 & 0.77 \\
Oleic (C18:1) & 85.13 & 5.51 & 13.75 & 9.57 & - \\
Linoleic (C18:2) & 7.75 & 1.27 & 2.23 & 1.92 & 0.28 \\
Arachidic (C20:0) & - & & - & - & 1.96 \\
Behenic (C22:0) & - & & - & 0.90 \\
\hline
\end{tabular}

Note: HOSO - high oleic sunflower oil; CO - coconut oil; PKO - palm kernel oil; PS - palm stearin; FHRO - fully hydrogenated rapeseed oil. Each value in the table represents the means of triplicate. 
TABLE 2. TRIACYLGLYCEROL (TAG) DISTRIBUTION OF STARTING OILS AND FATS

\begin{tabular}{|c|c|c|c|c|c|c|}
\hline \multirow{2}{*}{ Group } & \multirow{2}{*}{ TAG $^{*}$} & \multicolumn{5}{|c|}{ Samples } \\
\hline & & HOSO $(\%)$ & $\mathrm{CO}(\%)$ & PKO (\%) & PS $(\%)$ & FHRO $(\%)$ \\
\hline Chilled & OLL & 0.54 & - & - & - & - \\
\hline Chilled & OOL & 2.55 & - & - & - & - \\
\hline Chilled & $\mathrm{OOO}$ & 87.99 & - & - & 1.15 & - \\
\hline Chilled & PLO & - & - & - & 0.27 & - \\
\hline Room & $\mathrm{CaCC}$ & - & 0.24 & - & - & - \\
\hline Room & CaCLa & - & 2.22 & - & - & - \\
\hline Room & $\mathrm{LaOO}$ & - & 0.43 & 1.37 & - & - \\
\hline Room & POO & 5.24 & - & - & 1.50 & - \\
\hline Room & $\mathrm{LaLaO}$ & - & 0.71 & 3.12 & - & - \\
\hline Room & $\mathrm{SOO}$ & 3.00 & - & - & 0.43 & - \\
\hline Room & LaMO & - & 0.60 & 2.78 & - & - \\
\hline Room & MLP & 0.68 & - & - & - & - \\
\hline Room & PPL & - & - & - & 0.50 & - \\
\hline Body & CCLa & - & 17.20 & 7.70 & - & - \\
\hline Body & $\mathrm{MPO}$ & - & - & 0.44 & - & - \\
\hline Body & $\mathrm{LaPO}$ & & & 2.25 & & \\
\hline Body & CLaLa & - & 22.41 & 9.78 & - & - \\
\hline Body & POP & - & - & - & 9.38 & - \\
\hline Body & LaLaLa & - & 25.18 & 37.34 & - & - \\
\hline Body & LaLaM & - & 17.44 & 21.91 & - & - \\
\hline Body & POS & - & - & - & 6.37 & - \\
\hline Body & LaMM & - & 8.98 & 8.91 & - & - \\
\hline Heated & LaMP & - & 3.67 & 2.68 & - & - \\
\hline Heated & $\mathrm{LaPP}$ & - & 0.93 & 0.93 & - & - \\
\hline Heated & PPP & - & - & - & 80.41 & - \\
\hline Heated & SSP & - & - & - & - & 18.12 \\
\hline Heated & SSS & - & - & - & - & 81.87 \\
\hline [chilled] & & 91.08 &.- & - & 1.42 & - \\
\hline [room] & & 8.92 & 4.20 & 7.27 & 2.42 & - \\
\hline [body] & (lotal) & - & 91.21 & 88.63 & 15.75 & - \\
\hline [heated] & & - & 4.59 & 3.61 & 80.41 & 99.99 \\
\hline
\end{tabular}

Note: HOSO - high oleic sunflower oil; CO - coconut oil; PKO - palm kernel oil; PS - palm stearin; FHRO - fully hydrogenated rapeseed oil. Each value in the table represents the means of triplicate.

*Arranged fatty acid Ca - caprylic; C - capric, M - myristic; La - lauric; P - palmitic; S - stearic; O - oleic and L - linoleic.

In an ideal solubility mixture, the type of solvents (i.e. medium melting fat and liquid oils) does not give any different influence as long as those solvents do not interact with the high melting fraction during solidification. In this investigation, however, the melting thermogram showed some deviations on the onset of both binary mixtures at melting peaks of the high melting fraction. When the interval of melting peaks between the solute and the solvent was getting narrow such as in PKO mixtures (Figures $1 c$ and 2c), the deviations of the onset on the baseline were getting apparent. Some exothermic peaks, albeit few and small, were also observed on those deviated baselines. The presence of exothermic peak on the melting thermogram might indicate recrystallisation or polymorphic changes of the fat crystals. When molecular volume, shape and polymorph of the components in the mixture differ considerably yet those components have a comparable melting point, the eutectic system tends to take place (Timms, 1984).

Meanwhile, the shape of melting peaks of the high melting fractions at different molar ratios was generally comparable for both PS and FHRO binary mixtures. Lower molar ratios of hard fats gave smaller peaks with shifts of peak maximum to the lower temperatures. Table 3 showed that the type of solvents weakly influenced the experimental melting point $\left(\mathrm{T}_{\mathrm{m}}\right)$ of the hard fats. PS had lower experimental $\mathrm{T}_{\mathrm{m}}$ than FHRO at the interval temperature range from $63.12^{\circ} \mathrm{C}-63.68^{\circ} \mathrm{C}$ and from $70.12^{\circ} \mathrm{C}-70.30^{\circ} \mathrm{C}$ respectively, as influenced by the different type of solvents. Those values were comparable and in agreement with the melting point of pure systems. 
TABLE 3. THE EXPERIMENTAL MELTING TEMPERATURE $\left(\mathrm{T}_{\mathrm{m}}\right)$ AND HEAT OF FUSION $\left(\Delta \mathrm{H}_{\mathrm{f}}\right)$ OF HARD FATS IN DIFFERENT LOWER MELTING OILS

\begin{tabular}{lcc}
\hline \multirow{2}{*}{ Fat blend } & \multicolumn{2}{c}{ Melting properties } \\
\cline { 2 - 3 } & Melting temperature $\left({ }^{\circ} \mathbf{C}\right)$ & Heat of fusion $\left(\mathbf{k J ~ m o l}^{-1}\right)$ \\
\hline PS in MCT-oil & $63.12 \pm 0.07$ & $155.80 \pm 1.87$ \\
PS in HOSO & $63.30 \pm 0.00$ & $145.41 \pm 0.03$ \\
PS in PKO & $63.68 \pm 0.13$ & $115.44 \pm 2.81$ \\
FHRO in MCT-oil & $70.13 \pm 0.03$ & $187.91 \pm 1.26$ \\
FHRO in HOSO & $70.12 \pm 0.05$ & $174.51 \pm 1.49$ \\
FHRO in PKO & $70.30 \pm 0.02$ & $146.09 \pm 0.71$ \\
\hline
\end{tabular}

Note: MCT-oil - medium chain triglycerides oil; HOSO - high oleic sunflower oil; PKO - palm kernel oil; PS - palm stearin; FHRO - fully hydrogenated rapeseed oil.

Hildebrand equation is used in the calculation using data form differential scanning calorimeter (DSC) measurements. Each value in the table represents the means of triplicate.

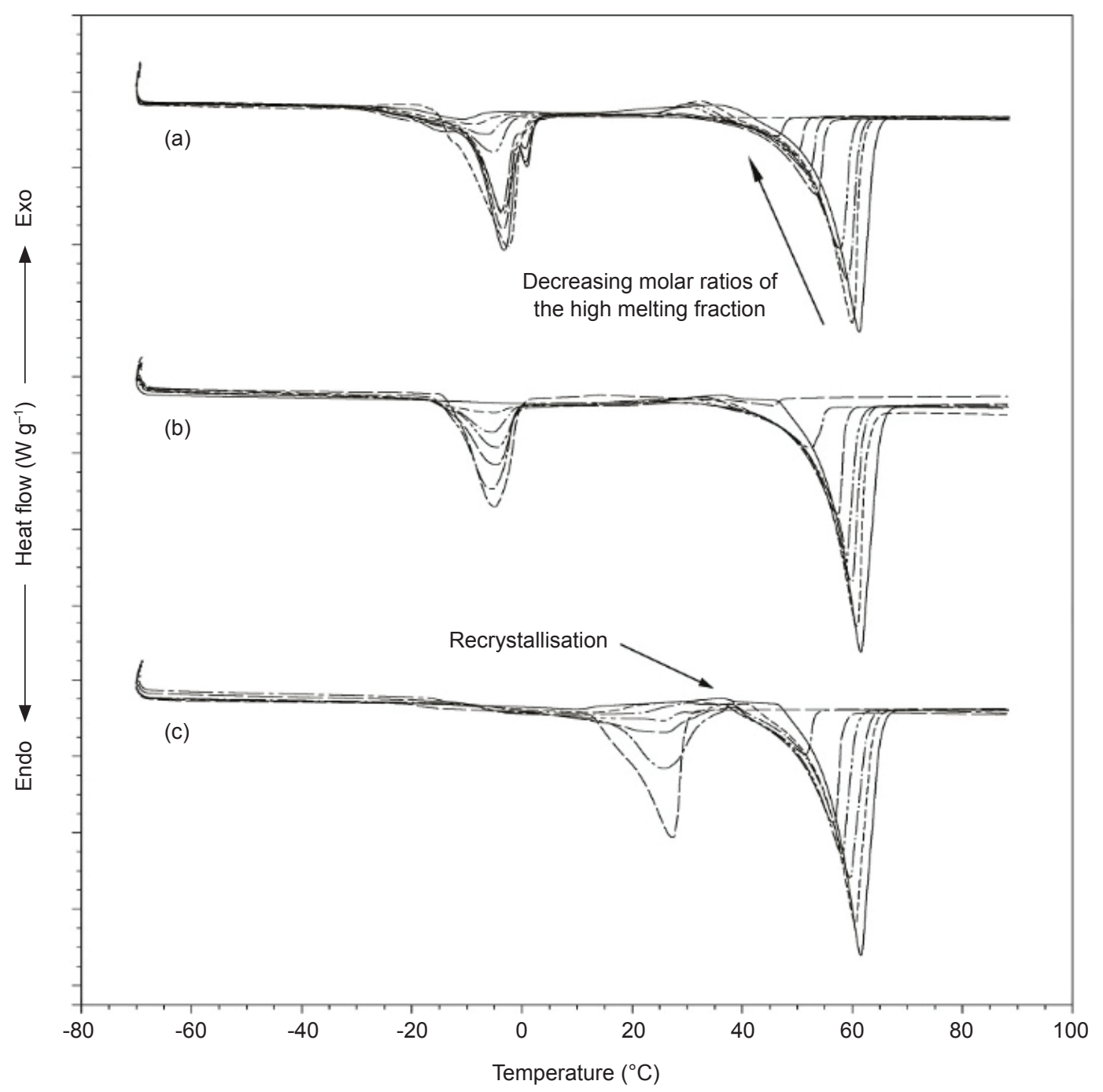

Note: DSC - differential scanning calorimeter; PS - palm stearin; MCT-oil - medium chain triacylglycerol oil; HOSO - high oleic sunflower oil; PKO - palm kernel oil.

Figure 1. DSC-melting behaviour of binary mixtures of PS in (a) MCT-oil, (b) HOSO, and (c) PKO. 


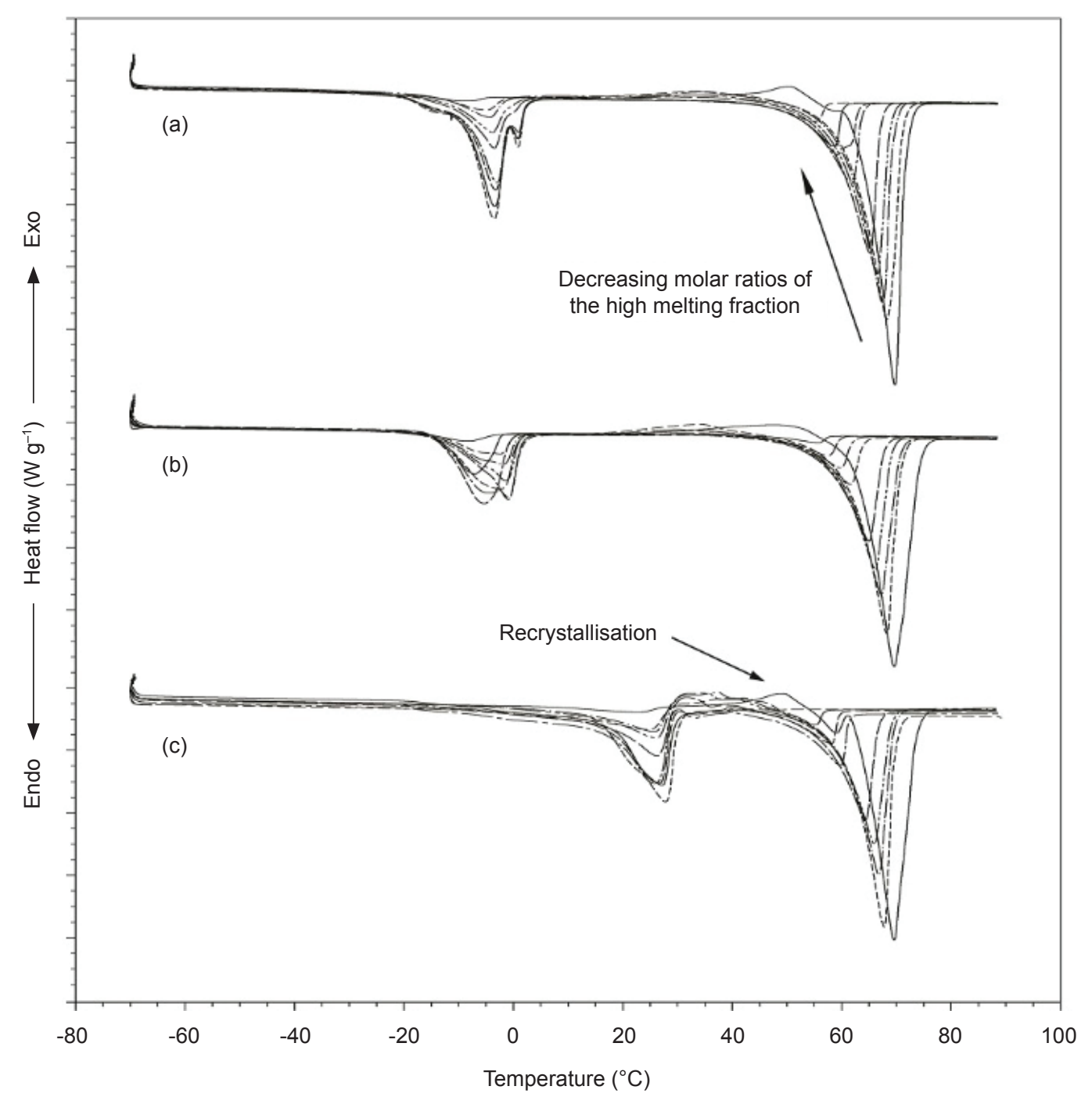

Note: DSC - differential scanning calorimeter; FHRO - fully hydrogenated rapeseed oil; MCT-oil - medium chain triacylglycerol oil; HOSO - high oleic sunflower oil; PKO - palm kernel oil.

Figure 2. DSC-melting behaviour of binary mixtures of FHRO in (a) MCT-oil, (b) HOSO, and (c) PKO.

On the other hand, the experimental heat of fusion $\left(\Delta \mathrm{H}_{\mathrm{f}}\right)$ of the hard fats was altered considerably depending on the type of solvents used in the binary mixtures. The liquid oils composed only of low melting TAG (i.e. MCT-oil and HOSO) slightly affected and resulted in comparable values of the experimental heat of fusion. The experimental $\Delta \mathrm{H}_{\mathrm{f}}$ of PS in MCT-oil and HOSO were 155.80 and $145.41 \mathrm{~kJ}$ $\mathrm{mol}^{-1}$, respectively while those of FHRO were 187.91 and $174.51 \mathrm{~kJ} \mathrm{~mol}^{-1}$, respectively. Nevertheless, PKO as the solvent in the binary mixtures caused considerably lower values of the experimental heat of fusions. The experimental $\Delta \mathrm{H}_{\mathrm{f}}$ of PS and FHRO with PKO as the solvent were 115.44 and $146.09 \mathrm{~kJ} \mathrm{~mol}^{-1}$, respectively. These lower values of experimental $\Delta \mathrm{H}_{\mathrm{f}}$ also coincided with stronger deviations on the onset of melting peaks of the hard fats. It should be taken into account that PKO contains some amount of high melting TAG such as 1-lauroyl-2-myristoyl3-palmitoyl-sn-glycerol (LaMP) and 1-lauroyl-2,3- dipalmitoyl-sn-glycerol LaPP (Table 2). These TAG could solidify together with the HMF of PS or FHRO thus modified its HMF composition, especially at lower molar ratios of the hard fats (meaning higher ratios of $\mathrm{PKO}$, thus, more LaMP and LaPP in the blends). As a result, different crystallisation behaviour and compensation of smaller $\Delta \mathrm{H}_{\mathrm{f}}$ of LaMP/LaPP brought the experimental $\Delta \mathrm{H}_{\mathrm{f}}$ of PS and FHRO lower.

In general, even though there were some indications of deviations from ideal solubility, the experimental $\mathrm{T}_{\mathrm{m}}$ values were still comparable and in agreement with the values of pure systems. Meanwhile, the values of experimental $\Delta \mathrm{H}_{\mathrm{f}}$ were also found to be comparable to those of pure systems when the solvents composed only of low melting TAG (e.g. MCT-oil and HOSO). As a comparison, the melting point and heat of fusion of tripalmitin (purity $>85 \%$ ) are $63.4^{\circ} \mathrm{C}$ and $150.5 \mathrm{~kJ} \mathrm{~mol}^{-1}$, respectively (Zhou and Hartel, 2006). 
Solubility of hard fats in the binary mixtures. The texture, mouthfeel, and melting point of plastic fats are influenced by the solubility of high-melting components in LMF of the fat blends. The melting and dissolution processes are characterised by melting enthalpy and enthalpy of dissolution of the mixtures. Hildebrand solubility equation (Equation 1) is theoretically valid when there is no enthalpy of dissolution during the process. However, it is acknowledged that fats and fat blends are a multicomponent TAG system of which some deviation from ideal solubility is commonly observed at some degrees (Humphrey and Narine, 2005).

In this investigation, the temperature of peak maximum on melting thermogram was chosen as the melting temperature $(\mathrm{T})$ of the blends for Hildebrand equation calculation. Meanwhile, the molecular weight of the samples was estimated from the average molecular weight of their fatty acid composition and it was used to convert the unit from gram to mole. The Hildebrand plot for the binary mixtures of two hard fats in three lower melting oils was then depicted in Figure 3. It was shown that the $\log$ value of the solubility [ $\mathrm{Ln}$ $\left(1 / x_{s}\right)$ ] generally had a linear relationship with the temperature $(1 / T)$, with a high coefficient of determination $\left(R^{2}\right)$ values. These linear relationships indicated that hard fats (PS or FHRO) might form an ideal solution with each of the lower melting oils. However, it was again noticed that hard fats diluted in PKO had solubility lines with stronger deviation from ideality compared to those of diluted in MCT-oil and HOSO, especially on the lower molar ratios. These deviations could be attributed to the changing in HMF composition of the hard fat and thus, their crystallisation behaviours. In general, the explanations were similar to the DSC melting behaviour as already discussed above.

\section{Solubility of High, Medium and Low Melting Fractions in Lauric Fat Blends}

Lauric fat blends were formulated as a ternary mixture composed of HMF, MMF and LMF. It was demonstrated in the simpler binary mixtures that hard fats were dissolved in either lauric or liquid oils. At the same time, the lauric oil was also shown to be dissolved in the liquid oils. Therefore, three distinct endothermal peaks were then observed on the DSC melting thermogram of lauric fat blends referring to those three fractions. Using the parameter of melting peaks of both HMF (H-group) and MMF (B-group), Hildebrand plot of FHRO lauric fat blends was constructed where the relationship between the solubility of both groups and temperature was illustrated by solubility curves as shown in Figure 4. We did not further elaborate the solubility of lauric fat blends with PS as the hard fat in this study as they had a similar pattern with that of FHRO lauric fat blends.

Lauric fat blends with FHRO as the hard fat formulated at constant $\mathrm{BH} 50$ with varied $\mathrm{H}$ from $2.5 \%-17.5 \%$ had two solubility curves representing the $\mathrm{H}$ and $\mathrm{B}$ groups. When the amount of H-group was increased, the amount of B-group was decreasing in order to compensate for the final sum of both groups at $50 \%$ in the blends. The changing of their ratios can be considered as the solubility of $\mathrm{H}$ and $\mathrm{B}$ groups in the LMF ( $\mathrm{C}$ and $\mathrm{R}$ groups, fixed at $50 \%$ ). In the case of $\mathrm{H}$-group, this HMF was dissolved both in MMF (B-group) and LMF (CR-group). As a result, the solubility of H-group was similar to that of the simpler binary mixture between HMF and lower melting components. It was found from the solubility curve that $\mathrm{H}$ group had a good linear relationship with $\mathrm{R}^{2}$ value of 0.9782 thus showed an ideal solubility. For B-group, this MMF dissolved in the LMF at the presence of H-group fat crystals. The lower amount of B-group resulted in more solid fat of H-group in the blends. The presence of solid fat might alter the crystallisation behaviour of B-group especially when the amount of both $\mathrm{H}$ and $\mathrm{B}$ groups was always changed following different formulation of the blends. The solidification of fats in the presence of other fat crystals could change the crystallisation behaviour of fat blends from homogenous to heterogeneous nucleation. It was eventually shown that stronger deviation from ideality was indicated on the solubility curve of MMF (B-group) at which the linear relationship between the composition [Ln $\left(1 / x_{s}\right)$ ] and temperature $(1 / T)$ had a lower $R^{2}$ value of 0.9401 .

On the other hand, FHRO lauric fat blends formulated at constant $\mathrm{H} 15$ with varied $\mathrm{BH}$ from $50 \%-70 \%$ had only one solubility curve in terms of H-group solubility in both MMF and LMF. Because the HMF was kept at $15 \%$ for all formulation, the solubility curve of H-group could not be drawn instead there was an average value of the correlation between the composition $\left[\operatorname{Ln}\left(1 / x_{s}\right)\right]$ and temperature $(1 / T)$. Meanwhile, the solubility of B-group in the LMF was influenced by the presence of $\mathrm{H}$-group fat crystals, now at a similar amount (15\% H-group) for all blends. Since the amount of fat crystal was set constant, they affected in the same way and gave comparable influences on the solubility of B-group in all blends. As a consequence, the solubility of B-group in lauric fat blends formulated at H15 was closer to ideality than that of blends formulated at BH50. Better linearity on the solubility curve of lauric fat blends formulated at $\mathrm{H} 15$ was shown by a higher $R^{2}$ value of 0.9954 .

\section{Solid Interaction in Lauric Fat Blends}

The interaction of solid fractions in the fat blends can be described by an isosolid diagram. 


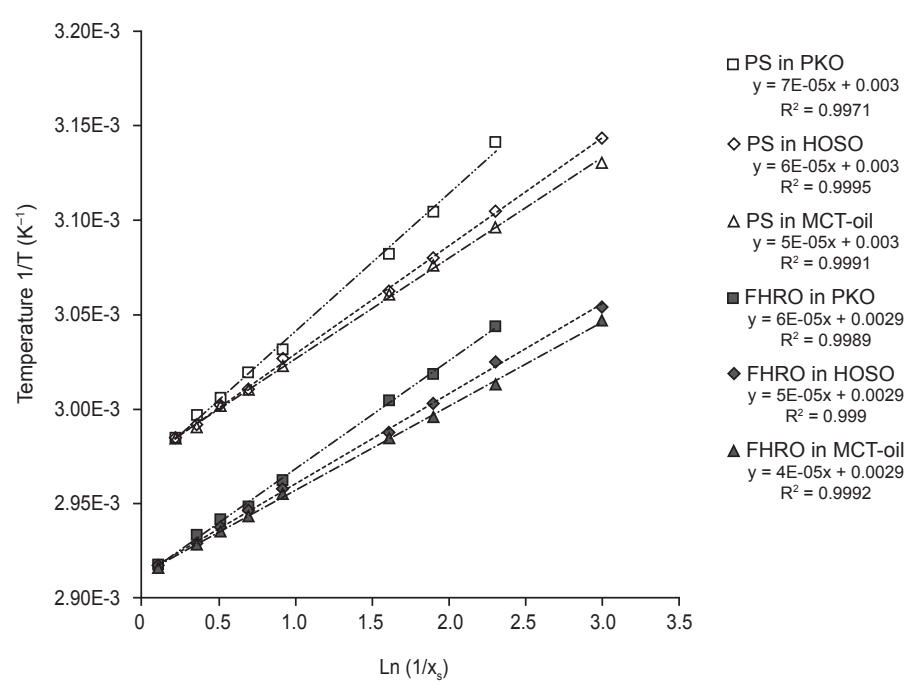

Note: PS - palm stearin; FHRO - fully hydrogenated rapeseed oil; PKO - palm kernel oil; HOSO - high oleic sunflower oil; MCT-oil - medium chain triacylglycerol oil.

Figure 3. Hildebrand plot of binary mixtures of high melting fractions in lower melting oils.

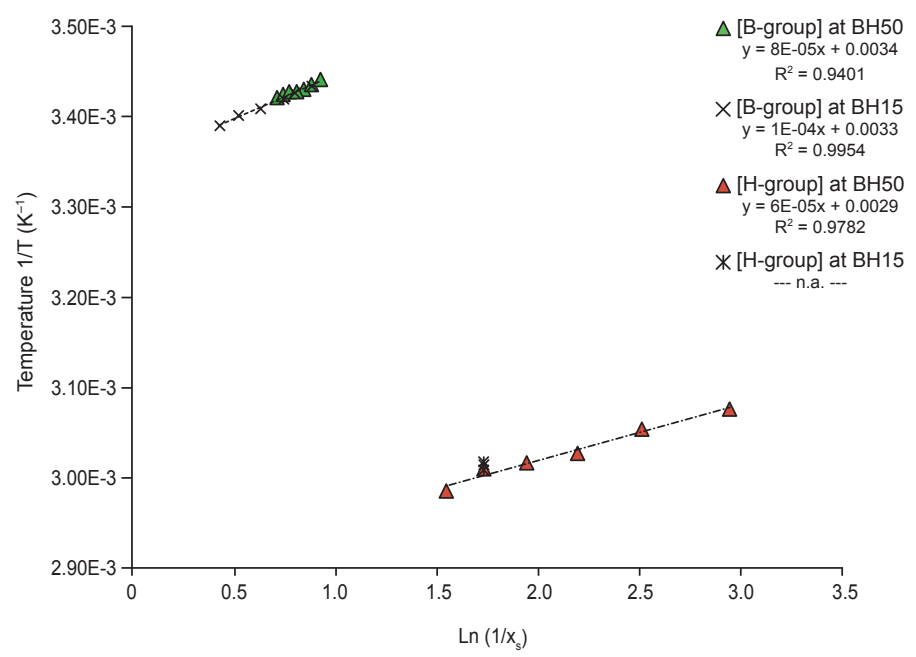

Note: B-group and H-group indicate the amount of medium melting fractions and high melting fractions in lauric fat blends, respectively. A formulation at $\mathrm{BH} 50$ or $\mathrm{H} 15$ refers to the fat blend composed of 50\% TAG from a combination of $\mathrm{B}$ and $\mathrm{H}$ groups or the fat blend composed of $15 \%$ TAG from $\mathrm{H}$ groups, respectively. Fully hydrogenated rapeseed oil (FHRO) was the hard fat added to lauric fat blends.

Figure 4. Hildebrand plot of FHRO lauric fat blends at different formulations.

This diagram contains isosolid lines representing the correlation between temperature and composition at constant SFC values. An isosolid line showed monotectic interaction as a straight horizontal line connecting two starting fats in fat blends. Meanwhile, eutectic interaction is indicated by compressed isosolid lines with melting temperatures below than those of the starting fats. Eutectic blends are usually undesirable as they had lower SFC than those of starting fats, showing that starting fats are not compatible (Timms, 1984; Lida et al., 2002). For solid interaction, we also discussed only FHRO lauric fat blends in this study as lauric fat blends with PS as the hard fat showed a comparable result.
The isosolid diagram of FHRO lauric fat blends formulated at constant BH50 was shown in Figure 5. In this formulation, all blends had a similar amount of LMF (C and $\mathrm{R}$ groups) at 50\%. Therefore, different $H$ values indicated different ratios of both $\mathrm{H}$-group and B-group in the constant amount of LMF (CR50). No depression was observed on the isosolid lines of lauric fat blends at this range of formulation (BH50 with varied $\mathrm{H}$ from 2.5\%-17.5\%) although a monotectic interaction was evident. Taking into account the previous binary mixture at which the solubility of hard fats in medium melting components had a slight deviation from ideality, this monotectic interaction seemed to coincide with its solubility curve. Thus, the effect 
of solid interaction was considered to be less pronounced than that of solubility. Moreover, the MMF formulated at BH50 was completely melted at approximately $20^{\circ} \mathrm{C}$ as indicated in the isosolid diagram. At any temperature higher than $20^{\circ} \mathrm{C}$, the SFC of the blends was apparently the function of $\mathrm{H}$-group ratios. It was shown that the higher ratios of H-group yielded higher SFC for isosolid lines, yet, with constant SFC less than $17.5 \%$.

Figure 6 showed the isosolid diagram of FHRO lauric fat blends formulated at constant H15. In this formulation, the amount of HMF in the blends was set constant at $15 \%$. Meanwhile, the amount of MMF (B-group) was varied against that of LMF (CRgroup) in order to fix the amount of both fractions at $85 \%$ in the blends. As the amount of HMF was fixed at $15 \%$, the isosolid lines at constant SFC up to 10\% displayed straight horizontal lines. These lines indicated the amount of fat crystal from H-group present in the blends at the temperature above the melting temperatures of both B-group and CR-group. Further, the isosolid lines at constant SFC values higher than $15 \%$ showed a monotectic interaction. The presence of solid fat (H-group) during B-group solidification seemed only imparting small influence as no depressions were noticed on those isosolid lines. The more closely spaced isosolid lines were also observed with the increment of B-group ratios indicating the dilution effect of B-group in CR-group (Ribeiro et al., 2009). The effect of solid interaction between HMF and MMF was thus considered to be less pronounced than the solubility effect of HMF / MMF in LMF similar to the previously formulated blends (BH50).

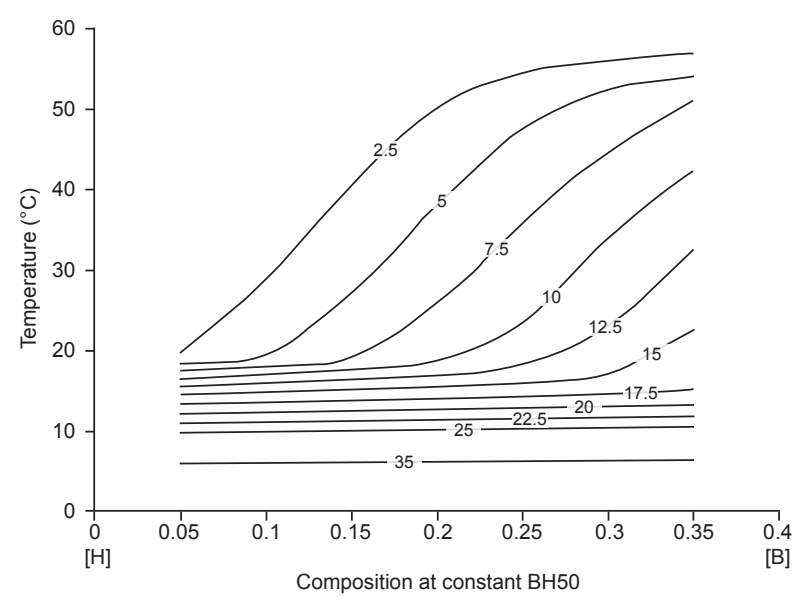

Note: A formulation at BH50 refers to the fat blend composed of $50 \%$ triacylglycerol (TAG) from a combination of $\mathrm{B}$ and $\mathrm{H}$ groups. Fully hydrogenated rapeseed oil (FHRO) was the hard fat added to lauric fat blends.

Figure 5. Isosolid diagram of FHRO lauric fat blends formulated at constant BH50.

\section{Hardness of Lauric Fat Blends}

The viscoelastic behaviour of plastic fats is mainly dictated by the structure of fat crystal network where the amount of solid fat, polymorphism and microstructure are known as the main influencing factors (Braipson-Danthine and Deroanne, 2004; Narine and Humphrey, 2004; Yanty et al., 2017). Hardness is commonly used to correlate that behaviour to the perceived texture of a plastic fat. It was shown that hardness of lauric fat blends was influenced by the amount of solid fat from both $\mathrm{H}$ and $\mathrm{BH}$ group. When the amount of TAG from $\mathrm{BH}-$ group was fixed at $50 \%$ (BH50) but that of H-group was varied (differ on the HMF ratio, $\mathrm{H} 2.5-\mathrm{H} 17.5$ ), hardness was generally found to be similar in the range 141-239 $\mathrm{g}$ and 164-308 $\mathrm{g}$ for PS and FHRO lauric fat blends, respectively (Figure 7). The different type of hard fats (PS, FHRO) also gave approximately comparable hardness. Nevertheless, an obvious pattern was observed at which hardness of FHRO lauric fat blends had higher values than that of PS lauric fat blends. The effect of both different ratios of HMF and hard fat types on hardness seemed to be small as the measurement was done at $15^{\circ} \mathrm{C}$ at which the amount of solidified fractions, both HMF and MMF, was thought to be similar (BH50).

Higher hardness values were found when the amount of TAG from BH-group was increased from $\mathrm{BH} 50$ to BH70 in lauric fat blends (Figure 8). At those blends, the HMF was set constant at 15\% (H15). Hardness corresponded well with the total amount of both HMF and MMF although the correlation was not linear. The variability in hardness could still arise from different crystal polymorph and network

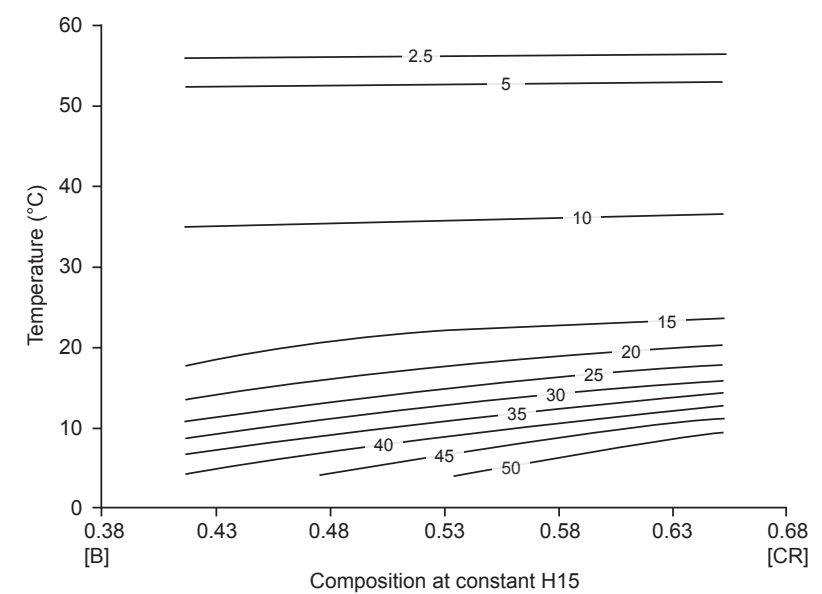

Note: A formulation at $\mathrm{H} 15$ refers to the fat blend composed of $15 \%$ triacylglycerol (TAG) from $\mathrm{H}$ group. Fully hydrogenated rapeseed oil (FHRO) was the hard fat added to lauric fat blends.

Figure 6. Isosolid diagram of FHRO lauric fat blends formulated at constant H15. 
structures (Braipson-Danthine and Deroanne, 2004) even when the system was revealed to have a monotectic interaction. In addition, higher hardness was also evident for all FHRO lauric fat blends compared to that of PS blends even so the values were mostly comparable. The higher concentration of the hard fats and longer carbon chain length of fatty acids on the TAG can result in fat blends with firmer texture (Zárubová et al., 2010; Manaf et al., 2019).

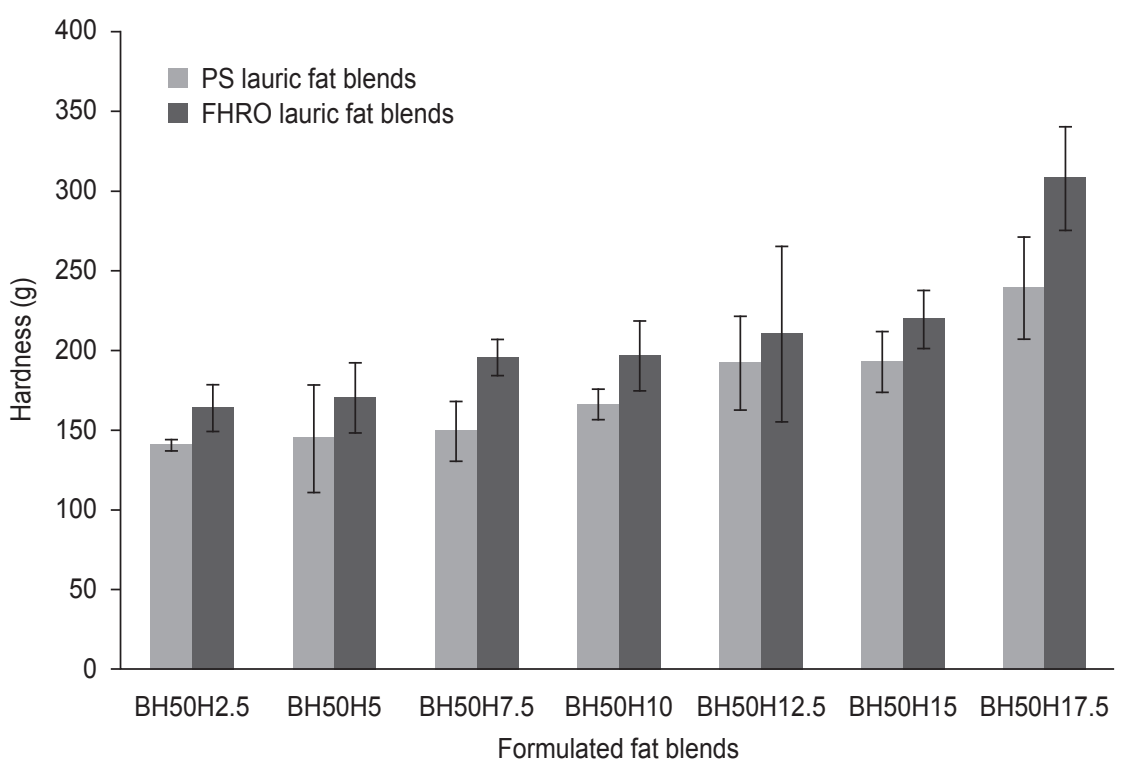

Note: Either palm stearin (PS) or fully hydrogenated rapeseed oil (FHRO) was the hard fat added to lauric fat blends. Meanwhile, a formulation at $\mathrm{BH} 50$ refers to the fat blend composed of 50\% triacylglycerol (TAG) from a combination of B and $\mathrm{H}$ groups. Lauric fat blends were formulated at constant $\mathrm{BH} 50$ and varied $\mathrm{H}$ from 2.5\%-17.5\% (BH50H2.5, $\mathrm{BH} 50 \mathrm{H} 5, \mathrm{BH} 50 \mathrm{H} 7.5, \mathrm{BH} 50 \mathrm{H} 10, \mathrm{BH} 50 \mathrm{H} 12.5$, BH50H15 and BH50H17.5).

Figure 7. Hardness of lauric fat blends formulated at constant BH50.

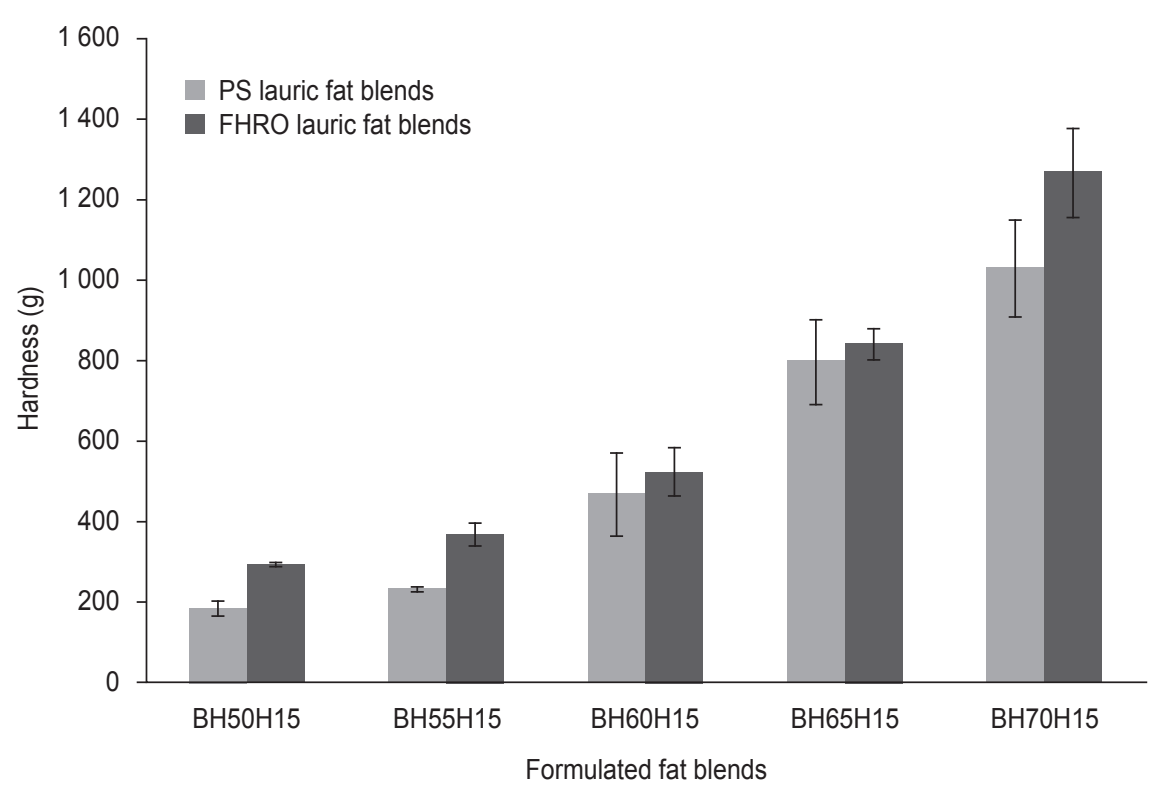

Note: Either palm stearin (PS) or fully hydrogenated rapeseed oil (FHRO) was the hard fat added to lauric fat blends. Meanwhile, a formulation at $\mathrm{H} 15$ refers to the fat blend composed of $15 \%$ triacylglycerol (TAG) from $\mathrm{H}$ group. Lauric fat blends were formulated at constant $\mathrm{H} 15$ and varied $\mathrm{BH}$ from 50\%-70\% (BH50H15, BH55H15, BH60H15, BH65H15 and BH70H15).

Figure 8. Hardness of lauric fat blends formulated at constant H15. 


\section{CONCLUSION}

This study demonstrated that the simpler binary mixtures could provide some basic information in describing the phase behaviour of more complex ternary lauric fat blends. It was shown in the binary mixture that hard fats (PS or FHRO) formed an ideal solubility in different liquid oils (MCT-oil or HOSO). However, the solubility of hard fats in the lauric oil was indicated by some deviation from ideality as PKO contained some amount of high melting TAG. They might, therefore, affect the composition of high melting fraction and its crystallisation behaviour of the hard fats. The experimental melting point $\left(\mathrm{T}_{\mathrm{m}}\right)$ and heat of fusion $\left(\Delta \mathrm{H}_{\mathrm{f}}\right)$ of the hard fats were comparable to those of pure system as ideal solubility was evidence. In the ternary lauric fat blends, the HMF formed an ideal solubility in the both MMF and LMF within the range of given formulations (at constant BH50 and H15). Meanwhile, the MMF also had a linear relationship on their solubility curves but with stronger deviation from ideality as affected by the presence of fat crystals from HMF. Further, the isosolid diagram of lauric fat blends generally exhibited monotectic behaviour. The influence of solubility, nevertheless, seemed to override the influence of solid interaction. In addition, the hardness of lauric fat blends was influenced by the total amount of HMF and MMF. Different type of hard fats gave small variability, yet FHRO lauric fat blends gave higher hardness values than those of PS blends. The solubility curves and isosolid lines, in general, provided valuable information about the interaction of high, medium and low melting fractions in the lauric fat blends. The pseudo-phase diagrams presented here could then be useful for improving the formulation of plastic fats, enriching the previous formulation based on the melting TAG group.

\section{ACKNOWLEDGEMENT}

The authors would like to thank the DirectorGeneral of MPOB for permission to publish this article.

\section{REFERENCES}

Braipson-Danthine, S and Deroanne, C (2004). Influence of SFC, microstructure and polymorphism on texture (hardness) of binary blends of fats involved in the preparation of industrial shortenings. Food Res. Int., 37: 941-948.

Chai, X; Meng, Z; Cao, P; Liang, X; Piatko, M; Campbell, S; Koon Lo, S and Liu, Y (2018). Influence of indigenous minor components on fat crystal network of fully hydrogenated palm kernel oil and fully hydrogenated coconut oil. Food Chem., 255: 4957.

Danthine, S (2012). Physicochemical and structural properties of compound dairy fat blends. Food Res. Int., 48: 187-195.

Drewnowski, A and Almiron-Roig, E (2010). Human perceptions and preferences for fat-rich foods. Fat Detection: Taste, Texture and Post Ingestive Effects (Montmayeur, J and Coutre, J eds.). CRC Press, Boca Raton, USA. p. 265-294.

Humphrey, K L and Narine, S S (2005). Lipid phase behavior. Fat Crystal Networks (Marangoni, A G ed.). Marcel Dekker, Inc., New York. p. 83114.

Lida, H N; Sundram, K; Siew, W; Aminah, A and Mamot, S (2002). TAG composition and solid fat content of palm oil, sunflower oil, and palm kernel olein blends before and after chemical interesterification. J. Am. Oil Chem. Soc., 79: 11371144.

Liu, C; Meng, Z; Cao, P; Jiang, J; Liang, X; Piatko, M; Campbell, S; Lo, S K and Liu, Y (2018). Visualized phase behavior of binary blends of coconut oil and palm stearin. Food Chem., 266: 66-72.

Manaf, Y N; Marikkar, J M N; Mustafa, S; Van Bockstaele, F and Nusantoro, B P (2019). Effect of three plant-based shortenings and lard on cookie dough properties and cookies quality. Int. Food Res. J., 26: 1795-1802.

Narine, S and Humphrey, K (2004). A comparison of lipid shortening functionality as a function of molecular ensemble and shear: Microstructure, polymorphism, solid fat content and texture. Food Res. Int., 37: 28-38.

Nusantoro, B P; De-Clercq, N; Anthierens, K and Dewettinck, K (2013). Changing the SFC profile of lauric fat blends based on melting group triacylglycerol formulation. J. Am. Oil Chem. Soc., 90: 1607-1619.

Nusantoro, B P; Xanthina, M; Kadivar, S; Yanty, N A M and Dewettinck, K (2016). Enzymatic interesterification of lauric fat blends formulated by grouping triacylglycerol melting points. J. Am. Oil Chem. Soc., 93: 1051-1062.

Nusantoro, B P; Yanty, N A M; Van-de-Walle, D; Hidayat, C; Danthine, S and Dewettinck, K (2017). Calculation procedure for formulating lauric and palmitic fat blends based on the grouping of 
triacylglycerol melting points. Grasas y Aceites, 68: e221.

O‘Brien, R D (2009). Fats and Oils: Formulating and Processing for Applications. CRC Press, Boca Raton, USA. 680 pp.

Podchong, P; Inbumrung, P and Sonwai, S (2020). The effect of hard lauric fats on the crystallization behavior of cocoa butter substitute. J. Oleo Sci., 69(7): 659-670.

Ribeiro, A P B; Grimaldi, R; Gioielli, L A and Gonçalves, L A (2009). Zero trans fats from soybean oil and fully hydrogenated soybean oil: Physicochemical properties and food applications. Food Res. Int., 42: 401-410.

Rombaut, R; De-Clercq, N; Foubert, I and Dewettinck, K (2009). Triacylglycerol analysis of fats and oils by evaporative light scattering detection. J. Am. Oil Chem. Soc., 86: 19-25.

Saberi, A; Chin-Ping, T and Oi-Ming, L (2011). Phase behavior of palm oil in blends with palm-based diacylglycerol. J. Am. Oil Chem. Soc., 88: 1857-1865.

Timms, R E (1979). Computer program to construct isosolid diagrams for fat blends. Chem. Ind., 7 (April): 257-259.

Timms, R E (1984). Phase behaviour of fats and their mixtures. Prog. Lipid Res., 23: 1-38.
Walstra, P (2003). Physical Chemistry of Foods. Marcel Dekker, Inc., New York. 832 pp.

Wesdorp, L H; van-Meeteren, J A; de-Jong, S; v.d.-Giessen, R; Overbosch, P; Grootscholten, P A M; Struik, M; Royers, E; Don, A; de-Loos, T; Peters, C and Gandasasmita, I (2005). Liquidmultiple solid phase equilibria in fats: Theory and experiments. Fat Crystal Networks (Marangoni, A G ed.). Marcel Dekker, Inc., New York. p. 481-710.

Yanty, N A M; Marikkar, J M N; Miskandar, M S; Van-Bockstaele, F; Dewettinck, K and Nusantoro, B (2017). Compatibility of selected plant-based shortening as lard substitute: Microstructure, polymorphic forms and textural properties. Grasas y Aceites, 68: e181.

Zárubová, M; Filip, V; Kšandová, L; Šmidrkal, J and Piska, I (2010). Rheological and crystalline properties of trans-free model fat blends as affected by the length of fatty acid chains. J. Food Eng., 99: 459-464.

Zhou, S; Zhang, F; Jin, Q; Liu, Y; Shan, L; Zhang, T; Zou, X and Wang, X (2010). Characterization of palm kernel oil, palm stearin, and palm olein blends in isosolid diagrams. Eur. J. Lipid Sci. Technol., 112: 1041-1047.

Zhou, Y and Hartel, R (2006). Phase behavior of model lipid systems: Solubility of high-melting fats in low-melting fats. J. Am. Oil Chem. Soc., 83: 505511. 